\title{
Mechanical influences in sinusoidal force measurement
}

\author{
Christian Schlegel, Gabriela Kiekenap, Holger Kahmann, Rolf Kumme
}

Physikalisch-Technische Bundesanstalt, Bundesallee 100, 38116 Braunschweig, Germany

\begin{abstract}
The paper describes mechanical influences which disturb a sinusoidal force calibration and hence have an influence on measurement uncertainty. The measurements are based on the application of a scanning vibrometer and the use of triaxial accelerometers. The measuring of many acceleration points on the top mass of the transducer makes it possible to obtain acceleration distributions from which a standard deviation can be derived; the triaxial accelerometer allows the observance of certain effects, like rocking modes, or other problems related to specific excitation frequencies of the force transducer. Both measurements can be related to each other. The rocking effects are discussed with FEM model calculations.
\end{abstract}

\section{Section: RESEARCH PAPER \\ Keywords: Dynamic force; sinusoidal excitation; laser vibrometer; rocking motion; triaxial acceleration; FEM modal analysis}

Citation: C. Schlegel, G. Kieckenap, B. Glöckner, A. Buß, R. Kumme, Mechanical influences in sinusoidal force measurement, Acta IMEKO, vol. 4, no. 2, article 10, June 2015, identifier: IMEKO-ACTA-04 (2015)-02-10

Editor: Paolo Carbone, University of Perugia, Italy

Received August 14, 2014; In final form February 12, 2015; Published June 2015

Copyright: @ 2015 IMEKO. This is an open-access article distributed under the terms of the Creative Commons Attribution 3.0 License, which permits unrestricted use, distribution, and reproduction in any medium, provided the original author and source are credited

Funding: This work was supported by EURAMET

Corresponding author: Christian Schlegel, e-mail: Christian.Schlegel@ptb.de

\section{INTRODUCTION}

Force measurement plays a major role in industrial processes, statically as well as dynamically. In the past a very versatile system of static force calibration was established, which last but not least can be recognized by the many high level force calibration laboratories and services around the globe. Nevertheless, most of the processes where force measurement is involved have a dynamic nature. So far only static calibrated force transducers also have been used in dynamic applications. The deviations in measurement associated with this may rise to the order of several percent and, especially in the vicinity of resonances, up to $10 \%$ to $100 \%$. To close this gap, some developments were carried out in the past to provide dynamic force calibration. These developments have also triggered a project currently running in the European Metrology Research Programme (EMRP), the "Traceable Dynamic Measurement of Mechanical Quantities", which includes, apart from a work package on dynamic force, also work packages on dynamic pressure, dynamic torque, the electrical characterization of measuring amplifiers and mathematical and statistical methods and modelling [2]. The investigation of the uncertainty contributions in sinusoidal force measurement is crucial for providing a reliable dynamic calibration of force transducers. A sinusoidal calibration is usually performed with an electrodynamic shaker system. Thereby, the force transducer, mounted on this shaker, is equipped with a top mass, and the acceleration on the surface of this mass as well as the force transducer signal are measured during the sinusoidal movement. A more detailed description of the whole calibration process can be found in [2].

\section{MEASURING AN ACCELERATION DISTRIBUTION}

A big advantage for the acceleration measurement is a scanning vibrometer which offers the opportunity to measure many points, e.g. on the whole surface of the mass block. By averaging the signals measured at these points, one can obtain realistic standard deviations, e.g. uncertainties. The dynamic behaviour during such a sinusoidal excitation depends on the kind of coupling of the top mass on the transducer as well as on the mounting of the transducer on the shaker table. In addition, of course, the internal mechanical structure of the transducer is of fundamental importance. One result of such a dynamic calibration is the dynamic sensitivity, which is the ratio between the force transducer signal and the measured dynamic force, which is the product of the acceleration of the top mass and its mass value. Due to the imperfect rigidity of the transducer, which depends on the internal structure, rocking modes may occur at certain frequencies. These rocking modes can be detected, for instance, from the uncertainty as a function 

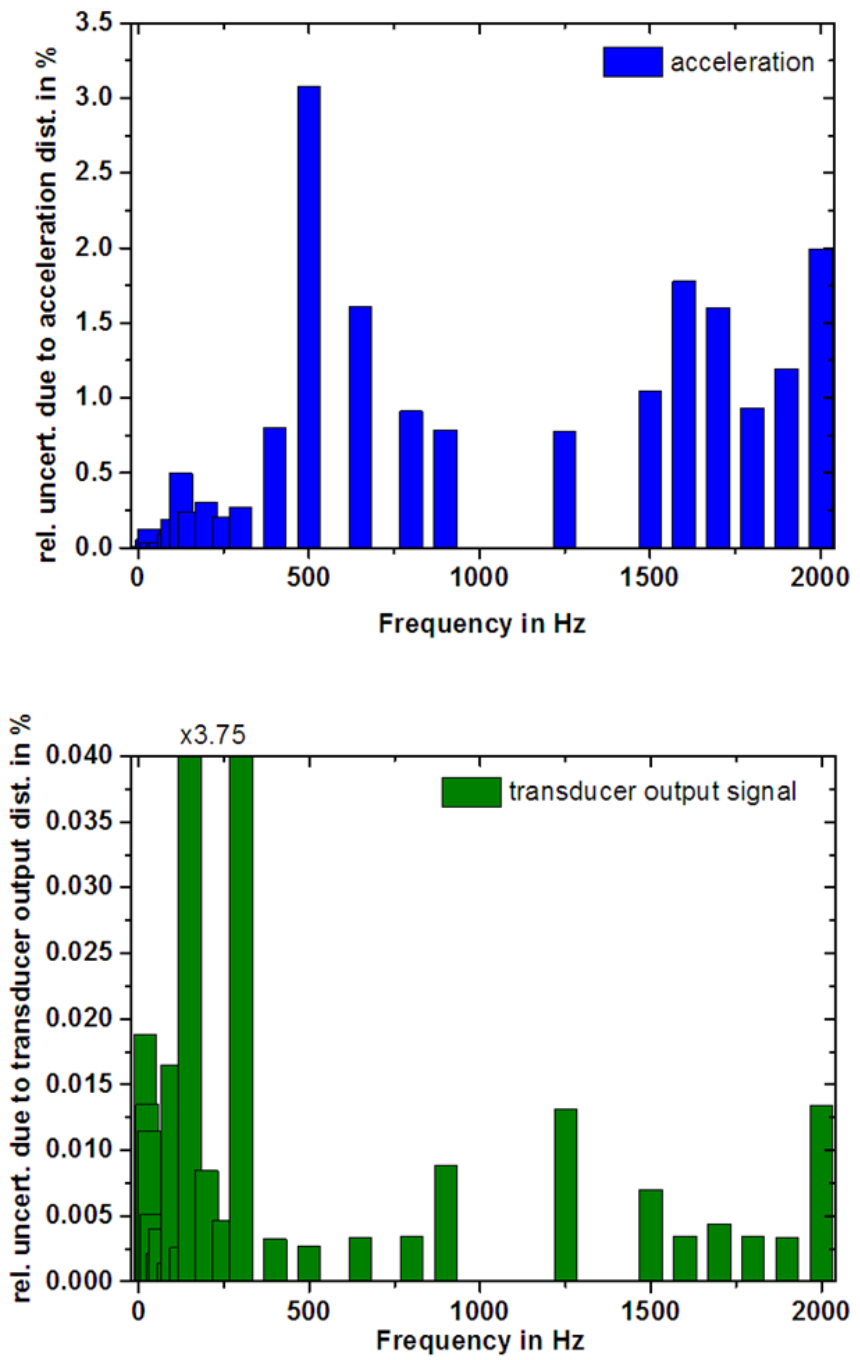

Figure 1. The figure shows the uncertainty of the measured acceleration on the top mass as well as of the force transducer signal. The uncertainty was obtained by averaging 62 acceleration measuring points and their 62 associated force signals at a certain frequency. It should be noted that the uncertainty of the vibrometer contributes to an additional $0.2 \%$ to $0.3 \%$ and the uncertainty of measurement of the force conditioning amplifier an additional $0.1 \%$ to $0.2 \%$. The two bars reaching $0.04 \%$ have to be multiplied by a factor of 3.75. Note, that the spatial acceleration distribution is not taken into account in this analysis; due to the quite small height of the mass block it can be neglected. In addition note the different scales of both plots.

of the frequency drawn for certain measuring channels. Figure 1 shows one example of such an analysis. In this special case the uncertainty of the acceleration shows several frequencies where the uncertainty is significantly higher than elsewhere. It should be noted, that this behaviour is not related to rocking modes of the shaker table, which was investigated elsewhere.

Surprisingly, this behaviour does not correspond to the synchronously measured transducer output signal. It seems that this special kind of force transducer is not so susceptible to interference from external influences. The origin of the relatively large uncertainties of the transducer output signal at $200 \mathrm{~Hz}$ and $300 \mathrm{~Hz}$ is unknown; obviously this increase has nothing to do with possible rocking modes.

In practice, one could conclude from such a measurement of the uncertainty distribution that it is advisable to avoid the regions where the transducer perhaps shows some irregularities.
It should be noted that the uncertainty given above is not the whole uncertainty because the contributions influenced by the measuring equipment are not considered. They yield, depending on the frequency range, an additional $0.3 \%$ to $0.5 \%$.

The scanning of the acceleration can be accompanied by triaxial acceleration measurements, where one can directly measure the transverse acceleration in certain directions. Combining the different methods may clarify the question of whether the increased uncertainty at certain frequencies can really be associated with rocking modes.

\section{TRANSVERSE ACCELERATION}

In order to investigate the possible rocking modes during the periodic excitation, the transverse acceleration was measured. For this purpose a special arrangement of four triaxial accelerometers on top of a test mass was chosen.

Figure 2 presents two pictures of the setup. The upper picture shows the force transducer equipped with an $8 \mathrm{~kg}$ test mass fixed with a mechanical adapter on the transducer. On top of the test mass the plate with the triaxial accelerometers can be seen. Besides the force transducer one can see one additional accelerometer mounted on the shaker table. The force transducer was an interface type with a nominal force range of $25 \mathrm{kN}$. Amplification of the force transducer output was realized with a DEWETRON conditioning amplifier.

The lower picture (Figure 3) shows the arrangement of the triaxial accelerometers on the plate which is mounted on the test mass.

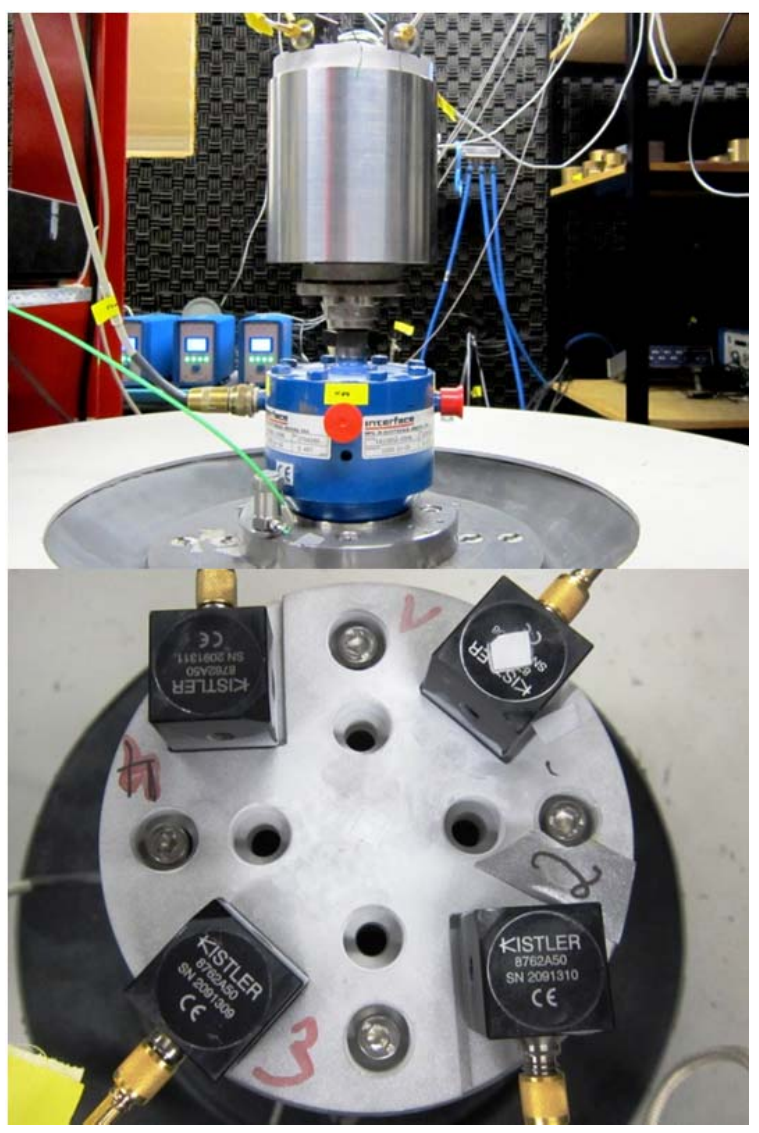

Figure 2. The upper picture shows the whole arrangement of the force transducer with the test mass, which was equipped with a special plate where four triaxial accelerometers have been mounted. The lower picture shows the plate with the four triaxial accelerometers. 




Figure 3 Schematic representation of the arrangement of the four triaxial accelerometers with their acceleration vectors in the transverse directions, $x$ and $y$. In addition there are four accelerations perpendicular to the shown plane, in the $z$ direction.

For a better fitting of the accelerometers on the plate, a certain area of the plate was milled out. The sensors themselves were screwed in place from the reverse of the plate.

The accelerometers were arranged in such a way that the $\mathrm{x}$ and y components have an equidistant separation of 45 degrees. The sensors themselves were from Kistler, type 8762A50, which have shear sensor elements that feature extremely low thermal transient responses and have a high immunity to base strain and transverse acceleration. An advanced hybrid charge amplifier is incorporated with a wide frequency range from $0.5 \mathrm{~Hz}-6 \mathrm{kHz}$. The acceleration range of the sensors is in a scale of $\pm 50 \mathrm{~g}$ whereby the peak limit is by $\pm 80 \mathrm{~g}$ and the sensitivity is $100 \mathrm{mV} / \mathrm{g}$, where $\mathrm{g}$ is the gravitational acceleration. The three outputs of each accelerometer were fed into the Kistler 5134B conditioning amplifier which also provides the power supply for the integrated amplifier of the sensor.

Figure 4 shows the acceleration measurement values according to the eight transverse directions as a function of the excitation frequencies. The behaviour can be quite well demonstrated in a kind of windmill plot. The length of the wings is proportional to the amplitude. The opening angle of the wings can be chosen arbitrarily, but is equal for all directions. On the scale on the left-hand side the amount of the acceleration amplitude in percent in relation to the z-amplitude is given. At first glance one can see that the transverse acceleration distribution changes with the frequency. If one observes at certain frequencies a rocking in the direction of $45^{\circ} / 225^{\circ}$, e.g. at $100 \mathrm{~Hz}$ and $1250 \mathrm{~Hz}$, the picture changes at frequencies of, e.g. $1750 \mathrm{~Hz}$ and $2000 \mathrm{~Hz}$, where the rocking occurs in the direction of $0^{\circ} / 180^{\circ}$. On the other hand, there are quite different amplitudes of the transverse acceleration as a function of frequency, which reach from a few percent at low frequencies up to $100 \%$ at high frequencies, related to the $z$-amplitude of the acceleration.

In Figure 5 the vector content of the transverse acceleration was calculated. According to the coordinate system, shown in Figure 5, the three vector components give one acceleration
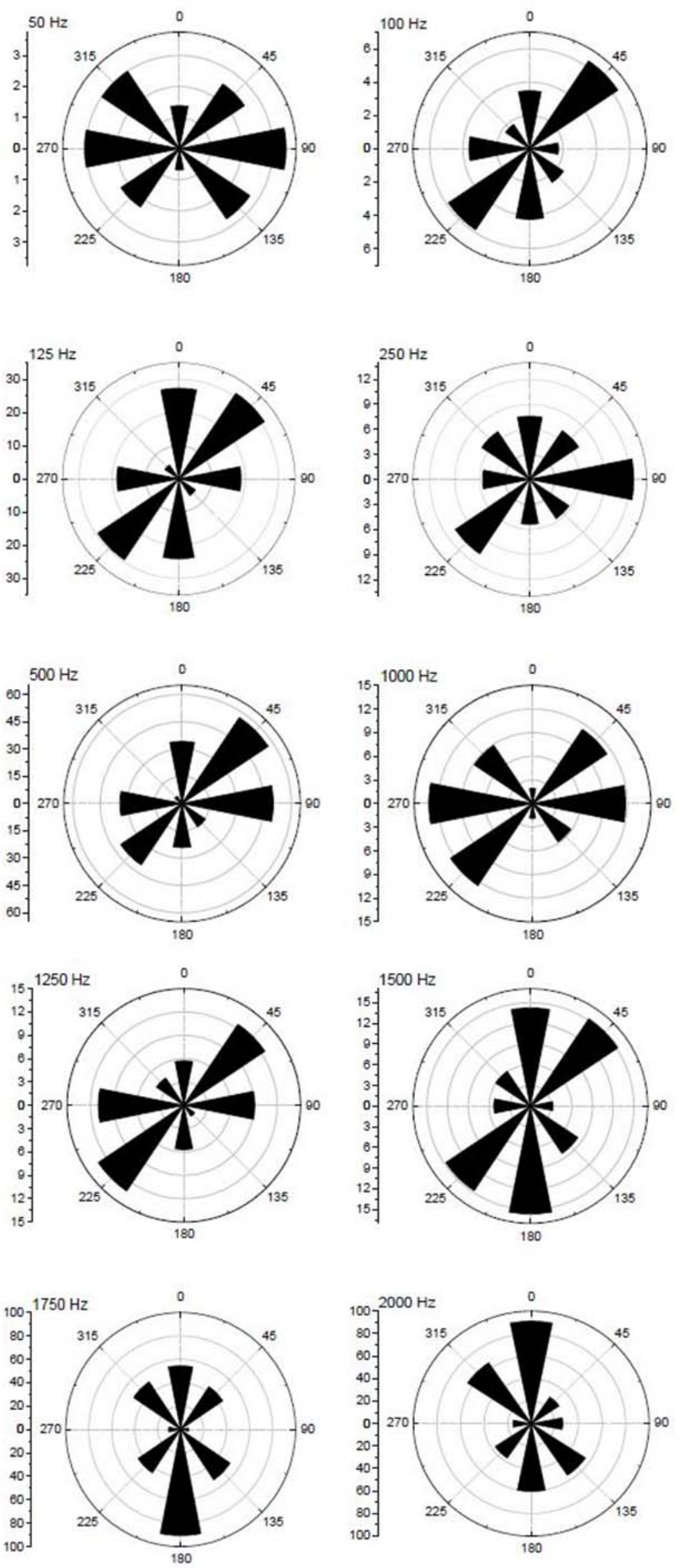

Figure 4. This figure shows the measured acceleration, observed in eight different directions at various frequencies, on top of the test mass. The scale on the left is the amplitude of the acceleration given as a percentage of the z-component of the acceleration. Note the widely-varying scales.

vector in space with a certain value, $\mathrm{R}$ and an angle of inclination, $\Theta$, in respect to the normal of the force vector, which is perpendicular to the upper surface of the top mass ( $\mathrm{z}-$ axis).

The corresponding projection of this vector on the xy-plane as a percentage of $\mathrm{R}$ and the angle, $\Theta$, is given in Figure 5 as a 


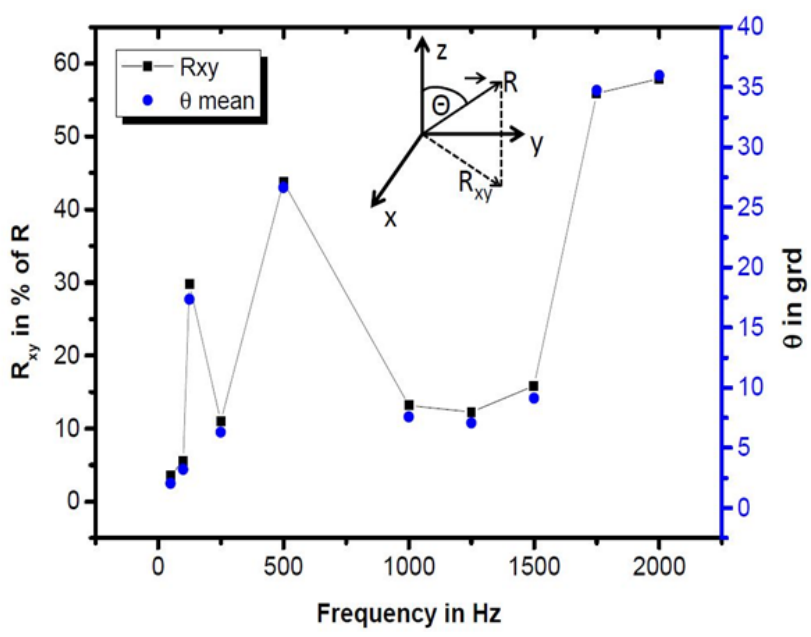

Figure 5. Vector components of the triaxial acceleration measurement. The amplitude of the transverse acceleration as well as the angle of inclination of the acceleration vector in respect to the vertical of the top mass surface (z-axis) as a function of the excitation frequency.

function of frequency. From the figure one can see that quite large rocking motions occur at certain frequencies. The question of what is the origin of such behaviour arises. The origin of the large transverse amplitude at $500 \mathrm{~Hz}$ is probably connected with a cross resonance of the transducer. The main resonance of the setup is around $930 \mathrm{~Hz}$. Experimentally, the uncertainties are large beyond the resonance frequency, which is also to be seen in this data, see e.g. Figure 1. If one compares both acceleration measurements, the scanning results according to Figure 1 and the transverse accelerations according to Figure 4 one can see a good correspondence at problematic frequencies, e.g. $500 \mathrm{~Hz}$. In summary, one can conclude that distinct frequencies with higher spatial variation of the top mass are often related to rocking modes of the experimental assembly which leads to higher uncertainties.

\section{FEM SIMULATION OF THE DYNAMIC SYSTEM}

To get more knowledge about the several dynamic modes which may happen during a periodical excitation an FEM Modal analysis was performed. In Figure 6 the investigated transducer can be seen as a FEM model in the upper left corner. The transducer is a shear force transducer, whereby the strain gauges are placed on the thin beams which are in between the holes. The inner part of the transducer is connected via a thread bolt to the outer environment, which provides the force initiation. In the case of the described periodical force measurement this is the additional mass block. By tension or compression of this inner part, the thin beams of the holes are distorted by higher stress, which are detected by the strain gauges. All following sub pictures show the behaviour of the transducer in connection with a $4 \mathrm{~kg}$ load mass. The frequencies of the dynamic modes are indicated below the figures, at which the dynamic modes occur.

At $261 \mathrm{~Hz}$ one has a first tilt resonance, where the mass block tilts relative to the transducer body. The red colour is thereby an indication of high stress, whereby the blue colour is a stress free situation. In the special case at $261 \mathrm{~Hz}$ that means, that these modes initiate additional stress in the mass block.
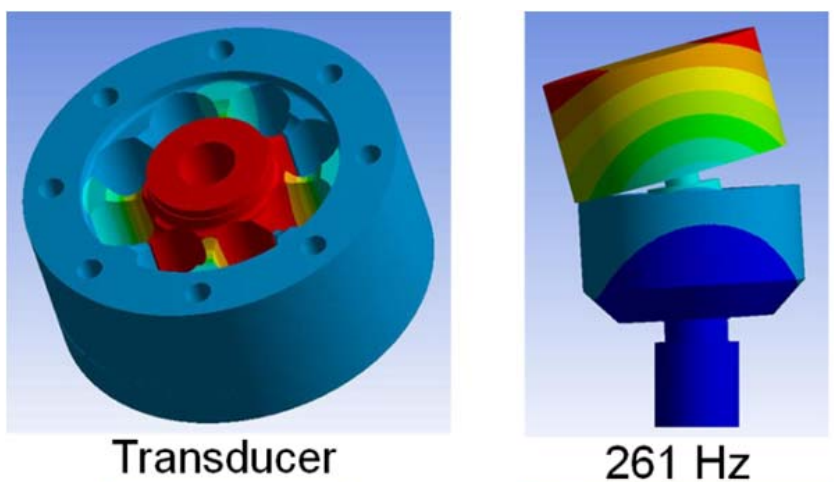

Transducer

$261 \mathrm{~Hz}$
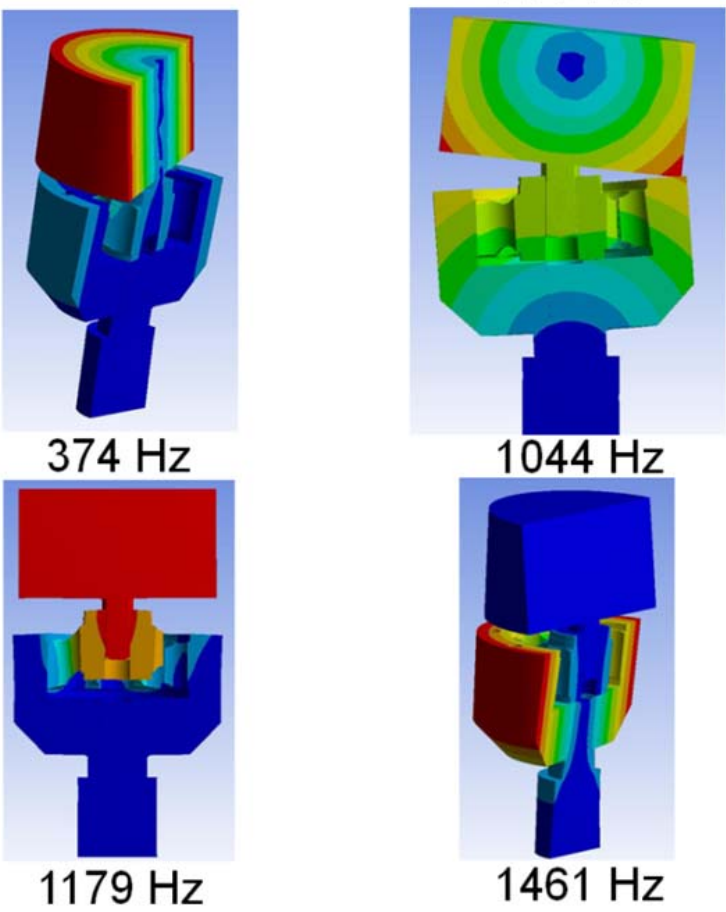

Figure 6. FEM model calculations of the force transducer equipped with a loading mass. Shown are the inner structure of the used transducer, upper left corner and the behaviour of the system at different modal frequencies The colours give the amount of stress level whereby red indicates high stress and blue no stress.

At $374 \mathrm{~Hz}$ one has a torsion vibration of the mass block against the transducer body.

Also here the additional stress is transferred to the mass block with the difference that, the stress rises from the inner to the outer diameter of the mass cylinder in cylindrical shells with different stress level.

At $1044 \mathrm{~Hz}$ there is a tilting of both, the mass block and the transducer with respect to each other. The amount of additional stress is more or less the same in the transducer as well as in the mass block. The tilting vibration is non concordant, which leads to the stress maximum at the outer part of the mass block on the bottom side.

At $1179 \mathrm{~Hz}$ there is the main system resonance, which is a vertical vibration of the mass block relative to the transducer. Here one can see a homogeneous stress level over the whole mass block.

At $1461 \mathrm{~Hz}$ there is a mode where the body of the transducer makes torsion in respect to the mass block. This mode is similar to the mode at $374 \mathrm{~Hz}$, with the difference, that the stress shells now occur in the transducer. 
Beyond $1500 \mathrm{~Hz}$ there are several other resonances which cannot be reached by the performed excitations.

\section{CONCLUSIONS}

This paper describes some of the mechanical influences which are present during a sinusoidal force measurement. In contrast to single point acceleration measurements, the measurement of the whole acceleration distribution on the surface of the top mass makes it possible to observe effects, like rocking modes or other mechanical deficiencies. Surprisingly, the effects seen by the acceleration signal are often suppressed in the force transducer output.

It could be shown by transverse acceleration measurements that the measured uncertainty distribution of the acceleration on the top mass has good correspondence with the transverse motions at least below the resonance frequency of the setup.

\section{ACKNOWLEDGEMENT}

The EMRP is jointly funded by the EMRP participating countries within EURAMET and the European Union.

\section{REFERENCES}

[1] European Metrology Research Programme (EMRP), http://www.emrponline.eu.

[2] C. Schlegel, G. Kieckenap, B. B. Glöckner, A. Buß, R. Kumme “Traceable periodic force calibration", Metrologia 2012, 49, $\mathrm{n}^{\circ} 3$, 224-235. 